EPJ Web of Conferences 31, 00037 (2012)

DOI: $10.1051 /$ epjconf/20123100037

(C) Owned by the authors, published by EDP Sciences - SIF, 2012

\title{
The elusiveness of multifragmentation footprints in 1-GeV proton-nucleus reactions
}

\author{
D. Mancusi ${ }^{1}$, A. Boudard ${ }^{1}$, J. Cugnon ${ }^{2}$, J.-C. David ${ }^{1}$, \\ T. Gorbinet ${ }^{1}$ and S. LERAY ${ }^{1}$
}

${ }^{1}$ CEA, Centre de Saclay, IRFU/Service de Physique Nucléaire, F-91191 Gif-sur-Yvette, France

${ }^{2}$ Fundamental Interactions in Physics and Astrophysics, University of Liège, allée du 6 août 17, bât. B5, B-4000 Liège 1, Belgium

\begin{abstract}
We use the tools of hybrid intranuclear-cascade/nuclear-deexcitation models to evaluate the sensitivity of several physical observables to the inclusion of a multifragmentation stage in the deexcitation chain and assess the need for a multifragmentation model in the quantitative description of $p+{ }^{56} \mathrm{Fe}$ and $p+{ }^{136} \mathrm{Xe}$ reactions at $1-\mathrm{GeV}$ incident energy. We seek clear signatures of multifragmentation by comparing different state-of-the-art de-excitation models coupled with intranuclear-cascade models and by focusing on discriminating observables such as correlations and fragment longitudinal-velocity distributions. None of the considered observables can be unambiguously interpreted as a multifragmentation footprint. The experimental data are best described as originating from sequential binary decays. However, no de-excitation model can reproduce the experimental longitudinalvelocity distributions from $1-\mathrm{GeV} p+{ }^{136} \mathrm{Xe}$.
\end{abstract}

\section{Introduction}

Multifragmentation is generally considered as the quasi-simultaneous breakup of highly-excited nuclear matter into clusters and unbound nucleons. Its

This is an Open Access article distributed under the terms of the Creative Commons Attribution License 2.0, which permits unrestricted use, distribution, and reproduction in any medium, provided the original work is properly cited. 

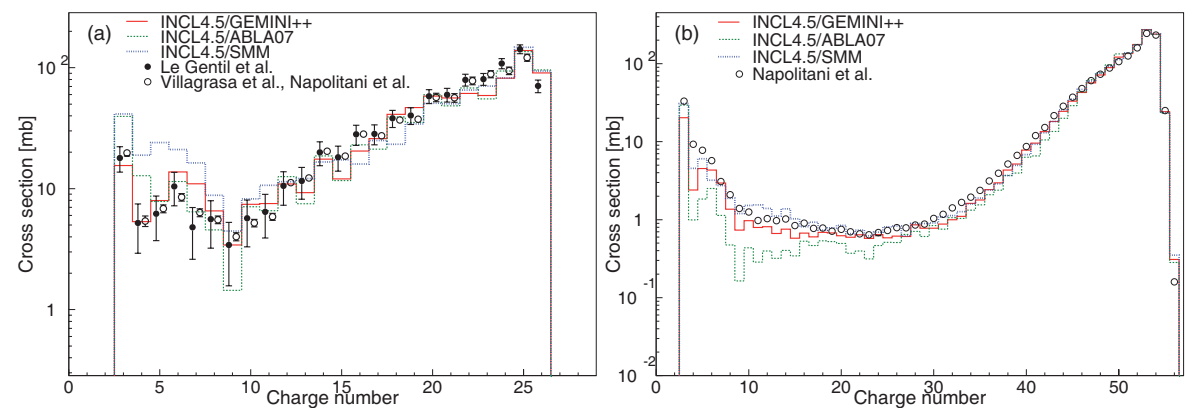

Figure 1: (Colour online) Inclusive residue-production cross sections for $1-\mathrm{GeV}$ $p+{ }^{56} \mathrm{Fe}(\mathrm{a})$ and $p+{ }^{136} \mathrm{Xe}(\mathrm{b})$, as a function of the nuclide charge. Experimental data from Refs. [2-5].

importance in nucleon-induced reactions is the subject of a long-standing discussion. While it is generally accepted that multifragmentation will eventually set in at high projectile energy, due to the increasing energy transfer from the projectile to the target nucleus, it is not yet clear whether and to what extent multifragmentation needs to be postulated for a reliable quantitative description of reactions around $1 \mathrm{GeV}$, a region which is most interesting for technical applications such as Accelerator-Driven Systems [1].

The goal of the present work is to identify possible signatures of multifragmentation by studying proton-induced reactions with the tools of coupled intranuclear-cascade/de-excitation models. We shall focus on the $1-\mathrm{GeV}$ $p+{ }^{56} \mathrm{Fe}$ and $p+{ }^{136} \mathrm{Xe}$ reactions, which have been the object of recent studies $[2-6]$.

All the calculations presented in this paper were performed using the Liège Intranuclear Cascade model (INCL4 .5 [7,8]), coupled with three different de-excitation models: ABLA07, [9], GEMINI++ [10,11] and SMM [12,13]. We limit ourselves to directing the reader to the relevant publications for details about the physics of the models. A more comprehensive discussion of the results of this study can be found in Ref. [14].

\section{Residue-production cross sections}

Figure 1 shows the predicted residue-production cross sections as a function of the nuclide charge, compared with the measurements obtained with the SPALADIN apparatus [3] or at the FRagment Separator (FRS) [2,4,5]. The experimental acceptance cuts have been taken into account.

We can observe that the three de-excitation models produce similar 
charge distributions, at least for charges $Z \gtrsim Z_{\text {target }} / 2$. In this sense, we observe that residue-production cross sections are rather insensitive to the de-excitation mechanism. However, we remark that de-excitation models present free parameters that can be adjusted to help reproduce the residueproduction cross sections. The GEMINI++ parameters connected with asymmetric fission were simultaneously fitted to the $p+{ }^{56} \mathrm{Fe}$ and $p+{ }^{136} \mathrm{Xe}$ residueproduction cross sections, among other data-sets [11]. Thus, fig. 1 can deceptively lead to underestimate the sensitivity of residue-production cross sections to the de-excitation model.

\section{SPALADIN correlations}

We now turn to the analysis of the model predictions for the SPALADIN $p+{ }^{56} \mathrm{Fe}$ data-set [3]. The goal of the SPALADIN experiment was to measure observables in coincidence for the $1-\mathrm{GeV} p+{ }^{56} \mathrm{Fe}$ reaction in inverse kinematics. These semi-exclusive measurements were obtained at the price of a more sophisticated setup and experimental analysis than typical inclusive experiments. Motivation for such measurements stems from the hope that more exclusive observables should help discriminating among different de-excitation models.

In this work, events generated by cascade/de-excitation models were filtered through a Geant4 transport calculation and analysed like the experimental data. A similar analysis, using older versions of the models, exists in the literature [3].

We analyse the decomposition of the residue-production cross sections (fig. 2). Experimental events were subdivided into five classes, according to the number of fragments $(Z \geq 3)$ and helium nuclei that were detected. Clearly INCL4.5/GEMINI++ and INCL4.5/ABLA07 yield the best description of the experimental data. Since GEMINI++ is a pure binary-decay model, and since ABLA07 predicts a very small multifragmentation cross section for this system, we conclude that it is possible to explain the event partitioning without invoking a multifragmentation model (such as SMM). This conclusion is consistent with Le Gentil et al.'s result [3]. However, the appraisal of SMM's results in the intermediate-mass-fragment region (IMF, defined as $3 \leq Z \leq$ 10 for the purpose of this paper) might be biased by its slight overestimation of the residue-production cross sections (see fig. 1). 

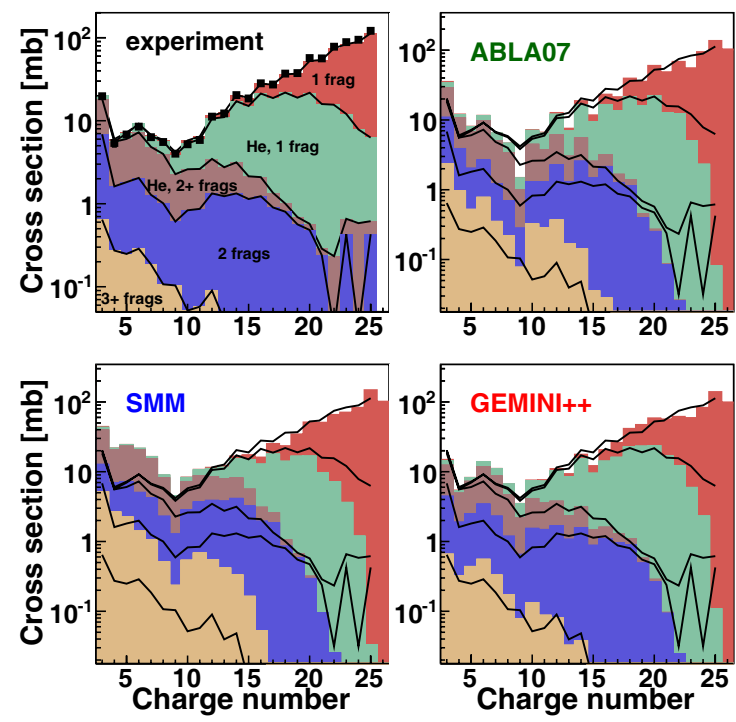

Figure 2: (Colour online) Cumulative partitioning of the $1-\mathrm{GeV} p+{ }^{56} \mathrm{Fe}$ cross section according to the number of observed helium nuclei and fragments $(Z \geq 3)$. Solid lines represent the experimental data and histograms represent the model predictions. Experimental data from Ref. [3].

\section{Longitudinal-velocity distributions}

Insight about the de-excitation mechanism can also be gained by examining the kinematics of the decay products. Sequential binary splits should produce kinematical patterns reminiscent of the decay barriers; multifragmentation, on the other hand, is expected to produce fragments with broad, structureless velocity distributions. We discuss here the velocity distributions measured in the context of the FRS experiments [4,6]. The emission velocities of fragments from the $1-\mathrm{GeV} p+{ }^{56} \mathrm{Fe}$ and $p+{ }^{136} \mathrm{Xe}$ reactions were measured using forward spectrometry techniques. Reactions were studied in inverse kinematics. Particles that satisfied the acceptance cut were identified by mass and charge and their longitudinal velocity (the component of the velocity along the beam axis) was measured. Refs. [4] and [6] report measured longitudinal-velocity distributions (LVDs) for several nuclides with $A \geq 6$.

We used our cascade/de-excitation tools to calculate the longitudinalvelocity distributions for the same nuclides. The acceptance of the experimental setup was approximately accounted for [14]. Figure 3 shows the calculated LVDs for some selected products of the ${ }^{56} \mathrm{Fe}+{ }^{1} \mathrm{H}$ and ${ }^{136} \mathrm{Xe}+{ }^{1} \mathrm{H}$ reactions. Each distribution is separately normalised to one. Note that the 


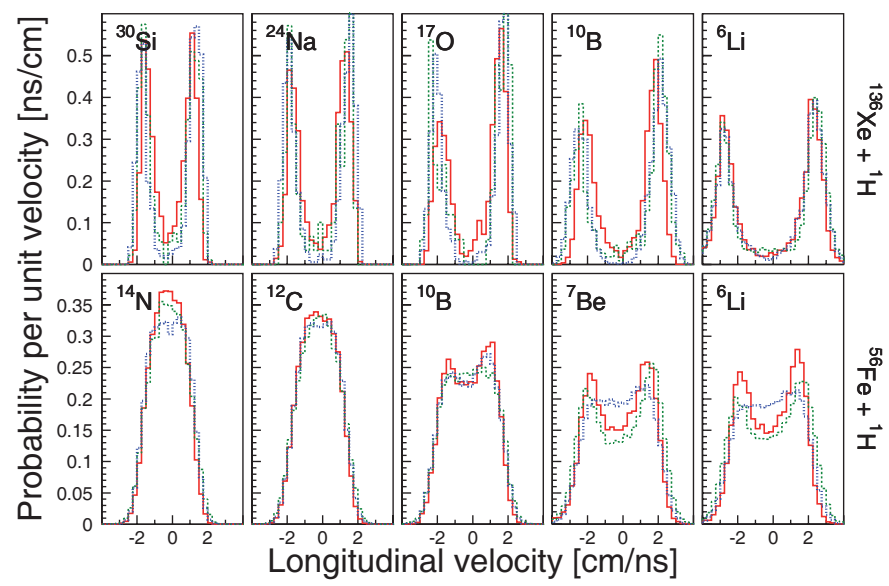

Figure 3: (Colour online) Calculated longitudinal-velocity distributions for some reaction products of the $1-A \mathrm{GeV}{ }^{56} \mathrm{Fe}+{ }^{1} \mathrm{H}$ and ${ }^{136} \mathrm{Xe}+{ }^{1} \mathrm{H}$ reactions, in the beam rest frame. Red solid line: INCL4 .5/GEMINI++; green dashed line: INCL4.5/ABLA07; blue dotted line: INCL4.5/SMM.

longitudinal velocities refer to the rest frame of the projectile, with the proton impinging with negative velocity. This choice was made for consistency with the experimental data $[4,6]$.

One observes that all models produce similar distributions for the heaviest IMFs. Only for the lightest IMFs and for the ${ }^{56} \mathrm{Fe}$ projectile can we observe differences in shape among the model predictions, with GEMINI++ and ABLA07 producing double-peaked distributions, whereas SMM yields flat distributions. These predictions should be compared with the measured distributions (fig. 10 in Ref. [4] and fig. 2 in Ref. [6]). In stark contrast with Napolitani et al.'s claims, we find that binary decay does not imply sharp Coulomb holes in the velocity distributions. Indeed, the shapes of the ${ }^{56} \mathrm{Fe}$ distributions seem to be best described by GEMINI++.

As displayed in fig. 3, all the predicted LVDs for ${ }^{136} \mathrm{Xe}+{ }^{1} \mathrm{H}$ show clear Coulomb holes. Surprisingly, even ABLA07 and SMM, that account for the contribution of multifragmentation, predict double-peaked LVDs that are very similar to GEMINI++'s, even for relatively heavy nuclides such as the Si isotope. No model seems to be able to account for the single-peaked component that clearly dominates the experimental LVDs for $Z \gtrsim 6$.

This unanticipated result clashes with the widespread belief that multifragmentation should yield single-peaked velocity distributions, especially for high fragment multiplicities. SMM accounts for the exact propagation of the hot multifragmentation products in their mutual Coulomb field, and is 
therefore supposed to yield reasonable predictions of the asymptotic velocities, if the initial conditions are realistic. Figure 3 demonstrates that this mechanism does not yield single-peaked LVDs. This suggests that most of the break-up configurations must be quasi-binary, with one or two large fragments completely dominating the Coulomb dynamics, possibly accompanied by nucleons and very light charged particles.

\section{Conclusions}

We have used the tools of coupled intranuclear-cascade and statistical-deexcitation models to search the $1-\mathrm{GeV} p+{ }^{56} \mathrm{Fe}$ and $p+{ }^{136} \mathrm{Xe}$ reactions for signatures of multifragmentation. Calculations indicate that the inclusion of a multifragmentation stage is not crucial for adequate prediction of residueproduction cross sections. We thus confirm the insensitiveness of this observable to the de-excitation mechanism. Comparisons with measured fragmenthelium correlations favour binary de-excitation models. We conclude that a multifragmentation model is not necessary for the description of inclusive and semi-exclusive observables. This does not mean that the presence of multifragmentation is ruled out in these reactions, but rather that binary decay can generate final states similar to those produced by multifragmentation models, at least close to the multifragmentation threshold.

Somewhat ambiguous conclusions can be drawn from the qualitative study of longitudinal-velocity distributions. We find that pure binary decay can well account for the distributions measured in $p+{ }^{56} \mathrm{Fe}$. On the other hand, none of the considered de-excitation models can explain the existence of the observed single-peaked component in $p+{ }^{136} \mathrm{Xe}$.

\section{References}

[1] Aït Abderrahim H. et al., Nucl. Phys. News, 20 (2010) 24.

[2] Villagrasa-Canton C. et al., Phys. Rev. C, 75 (2007) 044603.

[3] Le Gentil E. et al., Phys. Rev. Lett., 100 (2008) 022701.

[4] Napolitani P. et al., Phys. Rev. C, 70 (2004) 054607.

[5] Napolitani P. et al., Phys. Rev. C, 76 (2007) 064609.

[6] Napolitani P. et al., J. Phys. G: Nucl. Part. Phys., 38 (2011) 115006. 
[7] Boudard A. et al., Phys. Rev. C, 66 (2002) 044615.

[8] Cugnon J. et al., J. Korean Phys. Soc., 59 (2011) 955.

[9] Kelić A. et al., ABLA07 - towards a complete description of the decay channels of a nuclear system from spontaneous fission to multifragmentation, in Joint ICTP-IAEA Advanced Workshop on Model Codes for Spallation Reactions, page 181, Trieste, Italy, 2008, IAEA, Report INDC(NDC)-0530.

[10] Charity R. J., GEMINI: a code to simulate the decay of a compound nucleus by a series of binary decays, in Joint ICTP-IAEA Advanced Workshop on Model Codes for Spallation Reactions, page 139, Trieste, Italy, 2008, IAEA, Report INDC(NDC)-0530.

[11] Mancusi D. et al., Constraining statistical-model parameters using fusion and spallation reactions, in Proceedings to the FUSION11 conference, volume 17 of EPJ Web of Conferences, page 10003, Saint-Malo, France, 2011.

[12] Bondorf J. P. et al., Phys. Rep., 257 (1995) 133.

[13] Botvina A. S., Role of multifragmentation in spallation reactions, in Joint ICTP-IAEA Advanced Workshop on Model Codes for Spallation Reactions, page 131, Trieste, Italy, 2008, IAEA, Report INDC(NDC)0530 .

[14] Mancusi D. et al., Phys. Rev. C, 84 (2011) 064615. 